\title{
Initial Misdiagnosis of Cerebral Venous Thrombosis: A Case Report
}

\author{
Ram K. Alluria , Mehran Joseph Kashefi ${ }^{\mathrm{a}, \mathrm{b}, \mathrm{c}}$
}

\begin{abstract}
Cerebral venous thrombosis (CVT) is a rare condition with an incidence ranging from 2 to 4 cases per million people per year. Its presentation is highly variable and can include headaches, changes in vision, focal neurological deficits, seizures and coma. Treatment of CVT remains controversial, particularly regarding long-term anticoagulation. We report a case of a 35-year-old Asian male with a history of anti-thrombin III deficiency that was not adequately anticoagulated. He presented with an acute and progressively worsening left-sided headache and ipsilateral ear fullness. The patient was initially misdiagnosed with a migraine headache after computed tomography (CT) of the head was interpreted as normal.
\end{abstract}

Keywords: Venous sinus thrombosis; Cerebral venous thrombosis; Cerebral sinus thrombosis; Misdiagnosis; Neuroimaging; Anticoagulation

\section{Introduction}

Cerebral venous thrombosis (CVT) is a rare disorder with highly variable presentation that can complicate the recognition of this potentially fatal state [1-3]. Neuroimaging serves a critical role in the diagnosis of CVT and computed tomography (CT) is often the first imaging modality ordered; however, CT imaging of the head is not the most sensitive imaging technique, and in patients with hypercoagulable disorders, the threshold to order additional imaging studies

\footnotetext{
Manuscript accepted for publication September 20, 2013

${ }^{\mathrm{a}}$ David Geffen School of Medicine at UCLA, USA

${ }^{\mathrm{b}}$ Department of Medicine, West Los Angeles Veterans Administration, USA

${ }^{\mathrm{c} C o r r e s p o n d i n g ~ a u t h o r: ~ M e h r a n ~ J o s e p h ~ K a s h e f i, ~ D e p a r t m e n t ~ o f ~}$ Medicine, David Geffen School of Medicine at UCLA, 11301 Wilshire Blvd, Los Angeles, CA 90073, USA.

Email: mkashefi@mednet.ucla.edu
}

doi: http://dx.doi.org/10.4021/jmc1612e should remain low when CVT is high on the differential diagnosis [4]. The long-term management of patients with CVT and underlying hypercoagulable disorders remains controversial, but research over the past decade has helped form guidelines to facilitate anticoagulation decision-making.

\section{Case Report}

A 35-year-old man with a past medical history of anti-thrombin III (ATIII) deficiency and right-lower extremity deep vein thrombosis (DVT) presented to the emergency department complaining of a headache. The headache started suddenly three days prior. It was of severe intensity, progressively worsening, and bilaterally located along the vertex of his head. The pain radiated towards the left side of his neck as well. The patient also endorsed left ear discomfort, specifically described as "left ear fullness". Over-the-counter analgesics provided no symptomatic relief. Further history revealed the patient frequently suffered from migraine headaches and headaches associated with alcohol use; however, he stated this particular headache was unique. The pain was persistent and worsening when in the past his headaches spontaneously subsided over $24-48$ hours. The patient denied diplopia, photophobia, scintillations, tinnitus, vertigo, nausea, fevers, chills, dizziness, unsteady gait or recent trauma. Chart review revealed that the patient was prescribed life-long Coumadin for ATIII deficiency, but he had been noncompliant over the previous 3 weeks. His noncompliance was largely due to his recent history of drug abuse. The patient endorsed consuming one-fifth of whiskey per day and daily crystal methamphetamine use.

On physical exam his vital signs were stable and within normal limits. The patient's neck was supple and no focal tenderness was appreciated. His cardiovascular and respiratory exams were unremarkable. Examination of his skin revealed excoriated, erythematous, plaques located bilaterally across the antecubital fossa. Neurological exam revealed no ptosis, or nystagmus. Extra-ocular muscles were intact. The optic disc margins were sharp. Cranial nerves $2-12$ were normal. Motor, strength, sensation, reflex, and coordination exams were also within normal limits. Laboratory studies 


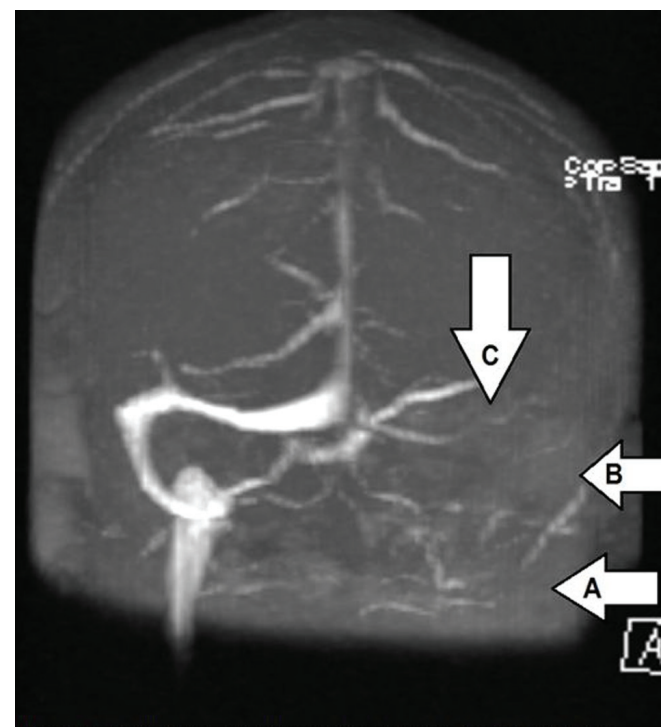

Figure 1. Magnetic resonance venogram (coronal view). Arrow A points to the absence of flow signal in the left internal jugular vein. Arrow $B$ points to the absence of flow signal in the left sigmoid sinus. Arrow $\mathrm{C}$ points to the absence of flow signal in the left transverse sinus.

were significant for a PT of 10.4, PTT of 30.5 and INR of 1.0. The initial CT head ordered was read as normal on the preliminary reading, but the final interpretation was concerning for venous sinus thrombosis. A follow-up CT venogram was consistent with CVT. CT venogram revealed a hyperdensity projecting along the left tentorium and extending into the left transverse sinus, left sigmoid sinus, and possibly the left jugular vein. Magnetic resonance (MR) venogram confirmed the aforementioned findings and additionally demonstrated absence of flow signal throughout most of the left internal jugular vein possibly associated with thrombosis or a congenitally diminutive left internal jugular vein (Fig. 1, 2). After imaging confirmed the diagnosis of CVT, the patient was started on Coumadin and bridged with low-molecularweight heparin (LMWH) until his INR became therapeutic. The patient was also counseled and provided with resources to manage his substance abuse, and he endorsed an interest in sobriety.

\section{Discussion}

CVT presenting with headaches is often misdiagnosed as common or classical migraines, tension headaches, subarachnoid hemorrhage, or CSF hypertension or hypotension [5]. With the advent of better imaging technology and increased awareness, the incidence of CVT is rising and currently estimated at 2 to 4 cases per 1 million, with the majority of these cases occurring in young adults, particularly females [2]. Headaches in CVT are mechanistically due

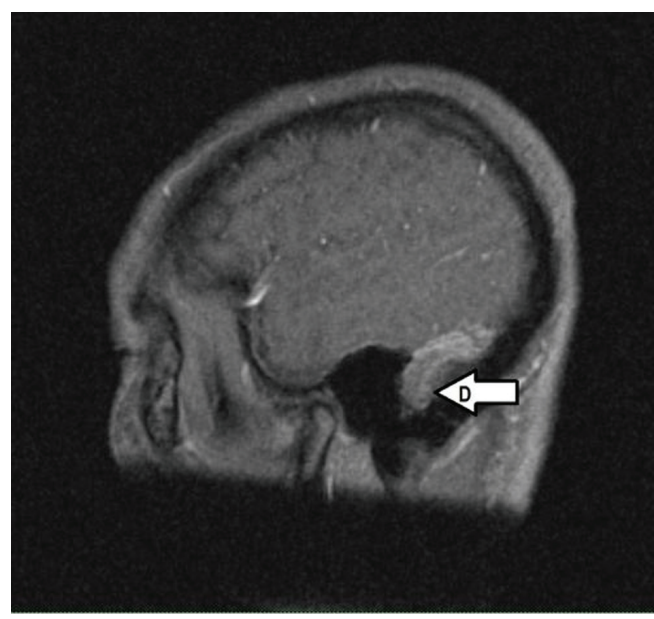

Figure 2. Magnetic resonance venogram (sagittal view). Arrow $\mathrm{D}$ points to the absence of flow signal in the left transverse sinus.

to intracranial hypertension which is theorized to occur by one of two pathways: thrombosis of cerebral veins resulting in localized ischemic edema or thrombosis of the major sinuses resulting in impaired CSF absorption [2]. The exact pathophysiological mechanism has not been elucidated, but numerous risk factors have been identified which may predispose a one to CVT [6]. The major risk factors include genetic and acquired thrombophilias, malignancies, hematologic abnormalities such as anemia or polycythemia, infections, and oral contraceptive use [7]. Over $80 \%$ of patients with CVT have 1 risk factor and over $40 \%$ have greater than 1 risk factor [7]. In addition to risk factor stratification of patients with suspected CVT, the likelihood of CVT is increased with particularly physical exam findings. Over $85 \%$ of patients with CVT present with headaches, often localized and of acute onset $[5,7]$. Other common physical exam findings include papilledema, visual loss, diplopia, aphasia, coma, paresis and seizures [7].

Laboratory studies of use in the diagnostic workup of CVT include a complete blood count, chemistry panel, prothrombin time, and activated partial thromboplastin time [8]. The role of D-dimer measurement has been found to be useful in numerous small studies, and current guidelines state D-dimer levels may be considered in a patient with low clinical suspicion for CVT; however, a normal D-dimer level is insufficient to exclude CVT in patients with strong clinical suspicion and further evaluation, such as cranial imaging, should be conducted [8]. Imaging of patients with suspected CVT is often a controversial topic and evidence-based guidelines suggest bypassing a non-contrast $\mathrm{CT}$ and ordering a T2weighted magnetic resonance imaging (MRI). Non-contrast $\mathrm{CT}$ in patients with CVT is only abnormal is approximately $30 \%$ of cases, leading to poor sensitivity [8]. Although MRI is recommended over CT, a negative MRI is insufficient to exclude CVT in a patient where clinical suspicion remains 
high. CT or MR venogram should be performed in these patients, but there is no evidence to suggest one venogram modality is more accurate than the other $[8,9]$.

Management of patients with CVT generally consists of initiating low molecular weight heparin or unfractionated heparin at full anti-coagulation doses with transition to vitamin $\mathrm{K}$ antagonists, with a target INR or 2.0 to 3.0 [8]. The duration of vitamin $\mathrm{K}$ antagonist treatment is 3 to 6 months in provoked CVT, and 6 to 12 months in unprovoked CVT [8]. In patients with a second episode of venous thromboembolism, indefinite anti-coagulation should be considered [8, 10]. Lastly, in patients with newly diagnosed CVT and no previous history of prothrombotic conditions, the following conditions should be tested for: protein $\mathrm{C}$ and $\mathrm{S}$ deficiency, anti-thrombin deficiency, anti-phospholipid syndrome, prothrombin G20210A mutation, and factor V Leiden mutation [8].

In the present case, the patient suffered from the classic acute onset, localized and progressively worsening headache. However, his history of recent drug abuse and migraine headaches made the underlying etiology of the headache less clear. His other diagnostic symptom was "left ear fullness", which may have been pulsatile tinnitus secondary to ipsilateral jugular sinus thrombosis [11]. His past medical history of a previous DVT and ATIII deficiency were also risk factors increasing the likelihood of CVT. Although CVT is an uncommon diagnosis, the patient's presenting symptoms and risk factors warranted further investigation and a noncontrast CT was ordered. Given the patient's risk factors and presentation, it may have been more prudent to bypass or follow-up the CT with an MRI [8]. Preliminary interpretation of the CT was normal, which is a common diagnostic pitfall in patients with underlying CVT. The misinterpretation of the CT led to a diagnosis of migraine headache, and put the patient at risk for CVT-related consequences such as cerebral ischemia and neurologic sequelae including seizures and coma. Fortunately, the final interpretation of the $\mathrm{CT}$ was concerning for CVT and the patient was contacted and admitted for further imaging and management. CT and MR venograms were ordered and the patient was started on LMWH and bridged to Coumadin. It was recommended that the patient continue indefinite Coumadin treatment given his prior history of a DVT and underlying ATIII deficiency.

\section{Competing Interest}

The authors declare that they have no competing interests.

\section{Authors' contributions}

All the above mentioned authors have contributed significantly to this case report. RA was involved in the direct care of this patient, and prepared the manuscript. MK was the attending internist responsible for the entire course of inhospital care for this patient and helped acquire the neuroimaging figures.

\section{Conflict of Interest}

The authors, their immediate family, and any research foundation with which they are affiliated have not received any financial payments or other benefits from any commercial entity related to the subject of this article.

\section{IRB}

Not applicable.

\section{Financial Disclosures}

None.

\section{References}

1. Agnelli G, Verso M. Epidemiology of cerebral vein and sinus thrombosis. Front Neurol Neurosci. 2008;23:1622.

2. Stam J. Thrombosis of the cerebral veins and sinuses. N Engl J Med. 2005;352(17):1791-1798.

3. Bousser MG, Ferro JM. Cerebral venous thrombosis: an update. Lancet Neurol. 2007;6(2):162-170.

4. Virapongse C, Cazenave C, Quisling R, Sarwar M, Hunter S. The empty delta sign: frequency and significance in 76 cases of dural sinus thrombosis. Radiology. 1987;162(3):779-785.

5. Agostoni E. Headache in cerebral venous thrombosis. Neurol Sci. 2004;25(Suppl 3):S206-210.

6. Filippidis A, Kapsalaki E, Patramani G, Fountas KN. Cerebral venous sinus thrombosis: review of the demographics, pathophysiology, current diagnosis, and treatment. Neurosurg Focus. 2009;27(5):E3.

7. Ferro JM, Canhao P, Stam J, Bousser MG, Barinagarrementeria F. Prognosis of cerebral vein and dural sinus thrombosis: results of the International Study on Cerebral Vein and Dural Sinus Thrombosis (ISCVT). Stroke. 2004;35(3):664-670.

8. Saposnik G, Barinagarrementeria F, Brown RD, Jr., Bushnell CD, Cucchiara B, Cushman M, deVeber G, et al. Diagnosis and management of cerebral venous thrombosis: a statement for healthcare professionals from the American Heart Association/American Stroke Association. Stroke. 2011;42(4):1158-1192. 
9. Leach JL, Fortuna RB, Jones BV, Gaskill-Shipley MF. Imaging of cerebral venous thrombosis: current techniques, spectrum of findings, and diagnostic pitfalls. Radiographics. 2006;26(Suppl 1):S19-41; discussion S42-13.

10. Schulman S, Granqvist S, Holmstrom M, Carlsson A, Lindmarker P, Nicol P, Eklund SG, et al. The duration of oral anticoagulant therapy after a second episode of venous thromboembolism. The Duration of Anticoagulation Trial Study Group. N Engl J Med. 1997;336(6):393398.

11. Waldvogel D, Mattle HP, Sturzenegger M, Schroth G. Pulsatile tinnitus--a review of 84 patients. J Neurol. 1998;245(3):137-142. 